\author{
Humanities and Social Sciences Letters \\ 2022 Vol. 10, No. 1, pp. 64-74. \\ $\operatorname{ISSN}(e): 2312-4318$ \\ $\operatorname{ISSN}(p): 2312-5659$ \\ DOI: $10.18488 / 73 . v 10 i 1.2934$ \\ (C) 2022 Conscientia Beam. All Rights Reserved. \\ check for
updates
}

\title{
COMMUNITY SOCIAL CAPITAL, FAMILY SUPPORT, CONFUCIAN PRO-SETBACK COPING AND GERIATRIC DEPRESSION: EVIDENCE FROM CHINA
}

\section{Yajun Qiao' \\ Zhong Wu $\mathrm{Li}^{2+}$}

\author{
${ }^{1,2}$ International College, National Institute of Development Administration, \\ Bangkok, Thailand. \\ 'Email:yajun.qia@stu.nida.ac.th Tel: 66922960318 \\ Email: zhongwu.li@nida.ac.th Tel: 66818476032
}

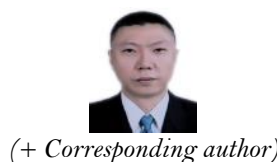

ABSTRACT
(+ Corresponding author)

\section{Article History \\ Received: 28 December 202 Revised: 31 January 2022 Accepted: 16 February 2022 Published: 4. March 2022 \\ Keywords \\ Community social capital Civic participation Social cohesion Family support Confucian pro-setback coping Geriatric depression.}

\begin{abstract}
It is important to explore the factors influencing geriatric depression in the context of aging. This research aimed to investigate the association between community social capital and depressive symptoms among the urban elderly in China, and the mediating role of family support and Confucian pro-setback coping in the impact of community social capital on geriatric depression. Data were collected via a community survey targeting 300 adults aged 60 and above living in urban communities in Chengdu, China, in 2021. SPSS and a structural equation model were used for the analysis of data and test the proposed hypothesis. The findings showed that civic participation and social cohesion were significantly related to geriatric depression. Family support and Confucian pro-setback coping played a mediating role or chain mediating role in the associations between civic participation and geriatric depression. This research has offered support for both direct and indirect effects of community social capital on geriatric depression. Promoting community-level civic participation and social cohesion, improving family support and Confucian pro-setback coping are seen to contribute positively to the management and intervention on geriatric depression.
\end{abstract}

Contribution/Originality: The paper's primary contribution is in identifying the direct effects of civic participation and social cohesion on geriatric depression and the mediating effects of family support and Confucian pro-setback coping on the relationship between civic participation and geriatric depression in China.

\section{INTRODUCTION}

As a form of mental disorder, geriatric depression seriously affects the health of the elderly. Geriatric depression has become a serious public health problem worldwide, as it leads to increased medical expenses and mortality (Vanoh, Shahar, Yahya, \& Hamid, 2016). The prevalence of depression in China among those aged 60 and above is much greater than in other East Asian countries; depression has been on the rise for the past 30 years, and it has become a serious problem (Lei, Xiaoting, John, Peng, \& Yaohui, 2014). A study has also shown that geriatric depression in China, between the years 2010 and 2019 affected $25.55 \%$ of the elderly population (Rong, Ge, Meng, Xie, \& Ding, 2020). In the context of an aging population, depression is most closely associated with unsuccessful aging (Golja, Ana, \& Voyko, 2020). Thus, to promote health and successful aging, public policies and programs related to the management of an aging population should focus on their mental health and depression (Dahany et al., 2014; Shen, 2014).

\subsection{Social Capital and Geriatric Depression}

The social capital theory argues that the characteristics of social organizations such as trust, norms and networks have a significant effect on promoting people's coordinated actions and improving social efficiency (Putnam, 1993). 
The term social capital refers to the resources and benefits obtained from individuals or groups, through connections with others (Rodgers, Valuev, Hswen, \& Subramanian, 2019). Social capital usually includes structural social capital (e.g., social engagement /participation) and cognitive social capital (e.g., social cohesion) (Nakagomi, Shiba, Hanazato, Kondo, \& Kawachi, 2020). Social capital may directly or indirectly affect depression in the elderly (Cao, Lu, Xudong, \& Chi, 2015).

Research has shown that increased community participation is positively correlated with a decrease in respondents' depression (Wang, Feng, Liu, \& Lu, 2020). Longitudinal studies have also shown that social involvement and social activities make positive contributions to the mental health of old people (Chiao, Li-Jen, \& Amanda, 2011). In relation to cognitive social capital, the elderly with low levels of interpersonal trust and reciprocity have a significantly higher rate of depression (Han et al., 2018; Li, Zhou, Ma, Jiang, \& Li, 2017). Social cohesion and reciprocity are also associated with lower levels of depressive symptoms (Nakagomi et al., 2020). Furthermore, Community-level social capital can also affect geriatric depression (Yamaguchi et al., 2019).

\subsection{Family Support and Geriatric Depression}

The social support theory holds that individuals feel loved and cared for when they are part of a supportive social network (Cobb, 1976). Social support plays an important role in affecting geriatric depression (Cao et al., 2015). Social support is primarily derived from family, friends and important others (Grey et al., 2020; Zimet, Suzanne, Gordon, Sidney, \& Karen, 1990). Compared with general social support, family support is more unique than all other forms of social support in maintaining good physical and psychological health (Kamen, Cosgrove, McKellar, Cronkite, \& Moos, 2011). Inadequate family support and lack of social activities can increase the risk of depression in the elderly (Cong, Ping, Daochun, \& Lin, 2015). Research has shown that more family support is related to less depression among rural older people. The influence of negative attitudes and geriatric depression in both urban and rural areas can be buffered by family support (Liu et al., 2020). In addition, A 23-year longitudinal study showed that higher family support is associated with lower levels of depressive symptoms, and faster recovery from depression (Kamen et al., 2011).

\subsection{Confucian Pro-Setback Coping and Depression}

Coping styles can positively affect geriatric depression (Trouillet, Gana, Lourel, \& Fort, 2009). Culture is regarded as a social determinant of mental health, and also influences people on how to cope with health problems (Campbell \& Long, 2014; Murray, 2015). Coping styles vary according to cultural background. As Chinese people are deeply influenced by Confucianism, exploring the impact of coping styles on geriatric depression from the perspective of Confucian culture can provide people with effective thinking methods to cope with setbacks and difficulties arising from traditional cultures (Li \& Hou, 2012). Confucian coping refers to the coping and thinking styles that Chinese people exhibit when facing difficulties and setbacks, which include thinking about fate, pro-setback and responsibility. Confucian coping can influence individual mental health and a high level of Confucian pro-setback coping is negatively correlated with depression (Li \& Hou, 2012; Lihua, Gui, Yanghua, Liqiong, \& Jian, 2017).

\subsection{Social Capital, Family Support, Coping and Geriatric Depression}

Social capital is directly associated with geriatric depression as well as social support. An individual's stock of resources (social capital) can also provide social support while improving self- efficacy. Social capital can buffer and contribute to coping with stressful life events by means of helping people obtain better resources (Campbell, Wood, \& Kelly, 1999). Cognitive social capital has a positive effect on the increase in social support (Rung, Symielle, William, Edward, \& Edward, 2017) and a higher level of community social capital can also provide more social support in the family field (Wu, Bill, \& Holly, 2012). Moreover, a high level of social participation not only provides more social support, but also fewer depressive symptoms in the elderly (Li, Jiang, Li, \& Zhang, 2018). Furthermore, Social support positively affects individual coping as social support can assist individuals in seeking appropriate coping approaches 
when facing pressure or problems (Thoits, 1986). Social support also affects individual quality of life through problemfocused coping strategies (Hadjicharalambous \& Demetriou, 2020; Shrestha et al., 2019). In addition, elevating the levels of coping and social support are conducive to preventive intervention among low-income, depressed elders (McDonald, Thompson, Perzow, Joos, \& Wadsworth, 2020).

However, there are few studies on social capital at the community level in China, and the measurement standards are rather chaotic. Thus, this study aimed to further explore the specific influence of community social capital on geriatric depression. This research has provided a theoretical framework to interpret how the community social capital of the elderly is related to geriatric depression in urban China, focusing on civic participation and social cohesion, while exploring the mediating role of family support and Confucian pro-setback coping between community social capital and geriatric depression. Therefore, this study hypothesizes that community social capital is negatively related to geriatric depression while family support and Confucian pro-setback coping are crucial factors linking community social capital to geriatric depression.

\section{METHODS}

2.1. Sample

A cross-sectional survey based on people aged 60 and above was carried out in Chengdu, which is representative of a Chinese city with an aging population. This study examined the association between community social capital, family support, Confucian pro-setback coping and geriatric depression. The population interviewed was defined according to the age of retirees in China in the past three decades (Feng, Yeung, Wang, \& Zeng, 2019); the elderly in China generally refer to those aged 60 and above. In order to ensure the validity of the questionnaire and data collection process, training was conducted for the recruited volunteer group. With the consent of the community staff, the research team organized the elderly to participate in the interviews in community senior activity centers. The eligible participants were community residents aged 60 years or older with cognitive skills. A face-to-face structured questionnaire survey was conducted among the elderly who voluntarily participated and met the required conditions. Data collection was carried out between April and July 2021, and a total of 300 valid questionnaires were collected.

\subsection{Measurement}

\subsubsection{Measurement of Geriatric Depression}

The Geriatric Depression Scale (GDS-5) is a five-item scale which is as effective as the 15-item GDS used for screening depression among the elderly in a community (Hoyl et al., 1999). The scale used in this study had 5 items, 1 of which was, "I often feel helpless". A five-point Likert scale was adopted for the measurement of the degree of agreement with a question or statement. The range was from 'strongly disagree' to 'strongly agree', and the corresponding scores ranged from 1 to 5 , with $\geq 2$ points being considered to demonstrate clinically relevant depressive symptoms (Ji et al., 2020).

\subsubsection{Measurement of Social Capital}

The community-level social capital scale adopted in this study aimed to assess the social capital of the elderly. Indicators of the scale included participation in activities, trust and reciprocity, community attachment and so forth (Saito et al., 2017). Many studies support the idea that social cohesion or cognitive social capital includes trust and reciprocity ((Fone et al., 2007; Han et al., 2018; Park, 2017). Therefore, two dimensions of civic participation (six items) and social cohesion (six items) were selected to measure the community-level social capital in this study. The respondents were required to respond to statements such as "I often attend activities carried out by hobby activity groups" and "I listen to my neighbours' concerns and complaints". As noted above, a five-point Likert scale was adopted for the measurement of the degree of agreement with these statements, which ranged from 'strongly disagree' to 'strongly agree', with the corresponding scores ranging from 1 to 5. 


\subsubsection{Measurement of Family Support}

Family support was evaluated by the Perceived Social Support Multidimensional Scale (MSPSS), a tool commonly used to measure social support, with good internal consistency (Zimet et al., 1990). Four items were adopted for measuring the degree of family support, comprising statements such as "My family really tries to help me". The fivepoint Likert scale was again adopted for measuring the degree of agreement with the question or statement.

\subsubsection{Measurement of Confucian Pro-Setback Coping}

Confucian pro-setback coping was evaluated through the Confucian coping scale (Li \& Hou, 2012). The scale included 4 items such as "I am not afraid of failure or frustration". The five-point Likert scale was adopted for the measurement of the degree of agreement with a statement. The range was from 'strongly disagree' to 'strongly agree', and the corresponding scores ranged from 1 to 5 .

\subsubsection{Socio Demographic Variables}

Socio demographic characteristics usually include gender, age, education level, living arrangements and economic income. Besides adults aged 60 and above, the socio-demographic factors considered in this study consisted of gender (male or female); education level (below high school/ technical secondary school or above high school/ technical secondary school); living arrangements (not living alone or living alone) and monthly income (<3365 yuan or $\geq 3365$ yuan). Among these factors, the monthly income level was based on the median per capita disposable income of Chinese urban residents in 2020 of 3365 yuan $(\$ 498.44)$ per month (National Bureau of Statistics of China, 2021). These factors affect the depressive symptoms of the elderly living in the community (Ji et al., 2020).

\subsubsection{Statistical Analysis}

SPSS24 and structural equation modeling (SEM) were adopted for examining the ways in which community social capital affects geriatric depression. The socio-demographic characteristics of the elderly sample were described based on the SPSS24 analysis results and SEM was used to evaluate the model. Before conducting the analysis, the fit of the hypothetical model was tested using the following indicators: comparative fit index (CFI), normed fit index (NFI), Tucker-Lewis index (TLI) and root mean square error of approximation (RMSEA). The values of CFI, NFI and TLI close to and greater than 0.9 show a good fit (Schumacker \& Richard, 2010). A value of less than 0.06 of root mean square error of approximation (RMSEA) value is usually considered to be a good model fitting data (McNeish \& Wolf, 2021).

\section{RESULTS}

\subsection{Sample Socio Demographic Features}

Table 1 shows the demographic features of respondents. In the sample, 51.667\% were males, and 48.333\% were females. In education, $77.333 \%$ of respondents had education levels of below high school or technical secondary school and $22.667 \%$ were at or above high school or technical secondary school levels.

Regarding the monthly income, $81.333 \%$ had an average monthly income of < 3365 yuan, and $18.667 \%$ had an average monthly income of $\geq 3365$ yuan. On living arrangement, $18.667 \%$ were living alone while $81.333 \%$ of respondents were not living alone.

\subsection{Survey Reliability and Validity}

The Cronbach' alpha value of the latent variable was between 0.869-0.915. The figures in Table 2 indicate that the items have good internal consistency. The factor loading of items ranged from 0.766 to $0.884(>0.5)$, the explanatory variation of latent variables ranged from 64.680 to $72.163 \%$ (> 50\%), the KMO value was between $0.815-0.912(>0.5)$ and Bartlett's test of sphericity was significant $(\mathrm{p}<0.001)$, reflecting good construct validity of the survey measures. 
Table 1. Socio Demographic features $(\mathrm{N}=300)$.

\begin{tabular}{|c|c|}
\hline & $\mathbf{N}(\%)$ \\
\hline \multicolumn{2}{|l|}{ Gender } \\
\hline Male & $155(51.667)$ \\
\hline Female & $145(48.333)$ \\
\hline \multicolumn{2}{|l|}{ Education level } \\
\hline$<$ High School/technical secondary school & $232(77.333)$ \\
\hline$\geq$ High School/technical secondary school & $68(22.667)$ \\
\hline \multicolumn{2}{|l|}{ Monthly income } \\
\hline$<3365 \mathrm{CNY}$ & $244(81.333)$ \\
\hline$\geq 3365 \mathrm{CNY}$ & $56(18.667)$ \\
\hline \multicolumn{2}{|l|}{ Living arrangement } \\
\hline Living alone & $56(18.667)$ \\
\hline Not living alone & $244(81.333)$ \\
\hline
\end{tabular}

Table 2. Analysis for reliability and validity.

\begin{tabular}{l|c|c|c|c}
\hline Construct & Items & Cronbach's a & Factor loading & Explained variance (\%) \\
\hline Civic participation & 6 & 0.915 & $0.816-0.859$ & 70.307 \\
\hline Social cohesion & 6 & 0.890 & $0.766-0.838$ & 64.680 \\
\hline Family support & 4 & 0.870 & $0.835-0.871$ & 72.163 \\
\hline Confucian pro-setback coping & 4 & 0.869 & $0.815-0.884$ & 71.821 \\
\hline Geriatric depression & 5 & 0.893 & $0.833-0.858$ & 71.483 \\
\hline
\end{tabular}

\subsection{Results from Structural Equation Modeling (SEM) Analysis}

To better understand the ways in which community social capital affects geriatric depression, structural equation modeling (SEM) was used to conduct a model fit test of the overall model and path analysis of the hypothesis model (see Figure 1). The model fitting was satisfactory: CFI $=0.989$, NFI $=0.954$, RFI $=0.919$, TLI $=0.987$ and RMSEA $=$ $0.028<0.06$. These indicators reflect a good fit between the model and the data.

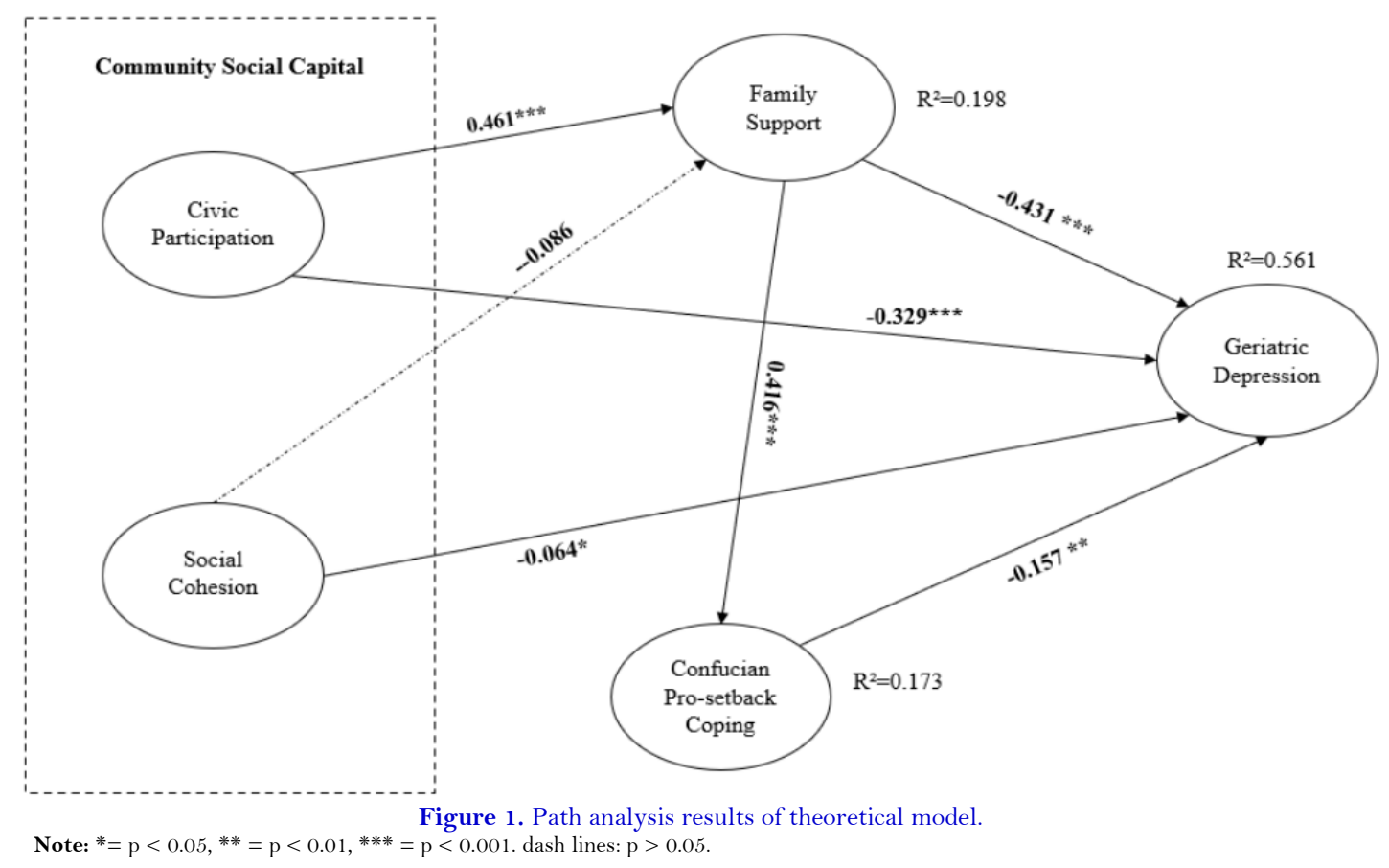

\subsubsection{Direct Effects of Community Social Capital on Family Support and Geriatric Depression}

Figure 1 and Table 3 present the outcomes of the path analysis. The results show that civic participation has a direct positive correlation with family support $(\beta=0.461, \mathrm{p}<0.001)$, which indicates that the higher the degree of civic 
participation, the better the degree of family support. The results also show that civic participation is negatively related to geriatric depression $(\beta=-0.329, \mathrm{p}<0.001)$ to a great degree. Therefore, the higher the degree of civic participation, the lower is the level of geriatric depression. For social cohesion, the results indicate that social cohesion is negatively related to geriatric depression $(\beta=-0.126, \mathrm{p}<0.05)$ to a great degree. Therefore, the stronger the social cohesion, the fewer are the symptoms of depression in the elderly. Meanwhile the relationship between social cohesion and family support is not significant $(\beta=-0.086, \mathrm{p}=0.176)$, as shown in Table 3 .

\subsubsection{Direct Relations between Family Support, Confucian Pro-Setback Coping and Geriatric Depression}

The results show that family support has a direct positive correlation with Confucian pro-setback coping ( $\beta=$ $0.416, \mathrm{p}<0.001)$, which indicates that more family support is conducive to helping the elderly cope with setbacks. Family support also has a negative correlation with geriatric depression $(\beta=-0.431, p<0.001)$ as shown in Table 3 . The results indicate that the more family support the elderly get, the less depression they suffer from. Furthermore, Confucian pro-setback coping has negative correlation with geriatric depression $(\beta=-0.157, p<0.01)$ as seen from Table 3. This result shows that the more Confucian pro-setback coping, the fewer are depressive symptoms seen in older adults. Figure 1 also shows that civic participation, social cohesion, family support and Confucian pro-setback coping have a total predictive power of $56.1 \%$ for geriatric depression.

Table 3. Results of direct effects

\begin{tabular}{l|c|c|c|c}
\hline \multicolumn{1}{c|}{ Path } & $\begin{array}{c}\text { Standardized } \\
\text { estimation }\end{array}$ & S.E. & C.R. & P \\
\hline Civic participation $>$ Family support & 0.461 & 0.064 & 6.766 & $* * *$ \\
\hline Civic participation $>$ Geriatric depression & -0.329 & 0.057 & -5.549 & $* * *$ \\
\hline Social cohesion $>$ Family support & -0.086 & 0.065 & -1.354 & 0.176 \\
\hline Social cohesion $>$ Geriatric depression & -0.126 & 0.053 & -2.503 & $*$ \\
\hline Family support $>$ Confucian pro-setback coping & 0.416 & 0.076 & 6.204 & $* * *$ \\
\hline Family support $>$ Geriatric depression & -0.431 & 0.068 & -6.446 & $* * *$ \\
\hline Confucian pro-setback coping $>$ Geriatric depression & -0.157 & 0.049 & -2.866 & $* *$ \\
\hline Note: $*$ p $<0.05, * *=p<0.01, * * *=p<0.001$. & & &
\end{tabular}

\subsubsection{Mediating Role of Family Support, Confucian Pro-Setback Coping in Community, Social Capital and Geriatric Depression}

In addition to the direct effects between variables, the conceptual framework of this study has also included some potential mediating effects of geriatric depression. The bias-corrected bootstrap sampling procedure was used to test these mediating effects. The bootstrapping procedure provides a more reasonable method to obtain specific indirect effects and can be adapted to most conditions (Bollen \& Robert, 1990; Preacher \& Hayes, 2008). At the 95\% confidence level, if the bias- corrected confidence interval (CI) does not contain zero, it means that the mediating effects are statistically significant. According to the results shown in Table 4, family support has a mediating effect between civic participation and geriatric depression. This result also shows that family support and Confucian pro-setback coping play a chain mediating role between civic participation and geriatric depression. However, family support and Confucian pro-setback coping have no mediating effect or multi-mediating effect on social cohesion and geriatric depression.

\section{DISCUSSION}

\subsection{Discussion of the Relationship between Community Social Capital and Geriatric Depression}

As a result of the poor social adaptation of the elderly, the influencing factors of geriatric depression are multifaceted. Low or lack of social capital usually affects the mental health of the elderly. The outcomes of this research show that the level of civic participation significantly affects geriatric depression. This conforms to past research 
findings where improvement in the level of community participation was seen to reduce depressive symptoms among the elderly (Landstedt, Ylva, Malin, \& Anne, 2016; Li et al., 2018; Wang et al., 2020). At the same time, research shows a significant association between social cohesion and geriatric depression (Murayama et al., 2015; Nakagomi et al., 2020). The findings of this study also reveal a significant association between social cohesion and geriatric depression.

Table 4. Results of mediating effects.

\begin{tabular}{|c|c|c|c|c|c|c|}
\hline \multirow[t]{2}{*}{ Path } & \multirow{2}{*}{$\begin{array}{c}\text { Point } \\
\text { estimation }\end{array}$} & \multicolumn{2}{|c|}{$\begin{array}{l}\text { Product of } \\
\text { coefficients }\end{array}$} & \multicolumn{2}{|c|}{$\begin{array}{c}\text { Bias-corrected } \\
\text { CI }\end{array}$} & \multirow[t]{2}{*}{ Result } \\
\hline & & SE & $\mathbf{Z}$ & Lower & Upper & \\
\hline $\begin{array}{l}\text { Civic participation } \rightarrow \text { Family Support } \\
\rightarrow \text { Geriatric depression }\end{array}$ & -0.199 & 0.033 & -6.030 & -0.271 & -0.140 & Supported \\
\hline $\begin{array}{l}\text { Social cohesion } \rightarrow \text { Family support } \rightarrow \text { Geriatric } \\
\text { depression }\end{array}$ & 0.037 & 0.029 & 1.276 & -0.018 & 0.096 & $\begin{array}{c}\text { Not } \\
\text { supported }\end{array}$ \\
\hline $\begin{array}{l}\text { Civic participation } \rightarrow \text { Family Support } \\
\rightarrow \text { Confucian pro-setback Coping } \rightarrow \text { Geriatric } \\
\text { depression }\end{array}$ & -0.030 & 0.013 & -2.308 & -0.062 & -0.009 & Supported \\
\hline $\begin{array}{l}\text { Social cohesion } \rightarrow \text { Family support } \rightarrow \text { Confucian } \\
\text { Pro-setback coping } \rightarrow \text { Geriatric depression }\end{array}$ & 0.006 & 0.005 & 1.200 & -0.002 & 0.019 & $\begin{array}{c}\text { Not } \\
\text { supported }\end{array}$ \\
\hline
\end{tabular}

\subsection{Discussion of the Association between Community Social Capital and Family Support}

In the process of human growth, social resources of individuals have an important impact on the evaluation of social support. The results of this study show that family support is significantly affected by civic participation, which conforms to the findings of Li et al. (2018) whereby a high level of social participation was seen to provide more family support. The findings of the current study also show the close relationship between improvement in civic participation and family support, as was shown in the study by Wang et al. (2020).

\subsection{Discussion of the Relationship between Family Support and Geriatric Depression}

As people enter old age and their bodies gradually weaken, social support can provide more care, especially close family support, which is greatly beneficial to the physical and mental health of the elderly. This study found that family support can efficiently decrease the symptoms of depression in the elderly. This finding conforms to that of Liu et al. (2020) who reported that family support can positively affect the depression levels of urban and rural elderly.

\subsection{Discussion of the Relationship between Confucian Pro-Setback Coping and Geriatric Depression}

People's positive coping with problems and difficulties can reduce the negative impact of stress events in life. Different coping styles which people choose are affected by different cultural backgrounds. Confucian coping, as a coping style adopted by Chinese people when facing difficulties and setbacks, has an important impact on Chinese people's behavior. The results of this study show that Confucian pro-setback coping has a significant impact on geriatric depression. This finding is consistent with the research results of Li and Hou (2012) and Lihua et al. (2017) who found that higher pro-setback thinking has a negative impact on depression.

\subsection{Suggestions for Social Capital Intervention, Improving Family Support and Coping}

This study has provided empirical research support for the management of aging and the prevention and control of geriatric depression. Due to the significant influence of community social capital on geriatric depression, the government needs to focus on the development of community capacity. Improving the environment and service quality of the community and creating more practical participation opportunities for the elderly will help to reduce the impact of geriatric depression (Haseda, Kondo, Takagi, \& Kondo, 2018). 
Furthermore, Chinese people attach great importance to the concept of family, thus family support also has a significant impact on geriatric depression. Family support intervention has a great effect on preventing and controlling geriatric depression (Wang \& Zhao, 2012). Besides family support, cultures may affect people's mental health. However, culture as an influencing factor is often overlooked. Confucian pro-setback coping can help with depression among the Chinese elderly. Therefore, it is necessary to enable more elderly people to draw positive energy from Confucian pro-setback coping to face difficulties, through community education and management. Managers should also guide and promote the role of Confucian culture in shaping Chinese people's mental health.

This study had some limitations. First, due to time constraints, the data collected was cross-sectional in nature, whereas longitudinal research can better understand the research concept and verify the direction of causality. Secondly, this study aimed to investigate the depressive symptoms of the elderly in urban communities, while there was a lack of investigation on the depression of the elderly in rural areas or in elderly care institutions. Moreover, the respondents in this study were generally healthy and some respondents may not have answered all the questions honestly. Therefore, they may not fully represent China's elderly population. Finally, this study also had some deficiencies in measurement. This study only involved family and social support, with no measurement and research on friend support and other important support areas. Therefore, these areas should be studied by future researchers.

\section{CONCLUSIONS}

This research has offered support for both direct and indirect effects of community social capital on geriatric depression. Promoting community-level civic participation, encouraging social cohesion, improving family support and Confucian pro-setback coping are deemed to be beneficial in alleviating the social impact of geriatric depression. Development and effective utilization of social resources and personal skills in the management of geriatric depression should be focused upon. The development of community capacity may increase the actual participation of the elderly and enhance social cohesion, thereby improving overall health.

Funding: This research is a part of the research project: Youth Foundation of Ministry of Education of China (Grant number: 19YJC630141).

Competing Interests: The authors declare that they have no competing interests.

Authors' Contributions: Both authors contributed equally to the conception and design of the study.

\section{REFERENCES}

Bollen, K. A., \& Robert, S. (1990). Direct and indirect effects classical and bootstrap estimates of variability. Sociological Methodology, 20, 115-140.Available at: https://doi.org/10.2307/271084.

Campbell, R. D., \& Long, L. A. (2014). Culture as a social determinant of mental and behavioral health: A look at culturally shaped beliefs and their impact on help-seeking behaviors and service use patterns of Black Americans with depression. Best Practices in Mental Health, 10(2), 48-62.

Campbell., C., Wood, R., \& Kelly, M. (1999). Social capital and health (pp. 15-16). London: Health Education Authority.

Cao, W., Lu, L., Xudong, Z., \& Chi, Z. (2015). Social capital and depression: Evidence from urban elderly in China. Aging and Mental Health, 19, 418-429.Available at: https://doi.org/10.1080/13607863.2014.948805.

Chiao, C., Li-Jen, W., \& Amanda, L. B. (2011). Social participation reduces depressive symptoms among older adults: An 18-year longitudinal analysis in Taiwan. BMC Public Health, 11, 1-9.Available at: https://doi.org/10.1186/1471-2458-1 1-292.

Cobb, S. (1976). Social support as a sediator of life stress. Psychosomatic Medicine, 38(5), 300-314.Available at: https://doi.org/10.1097/00006842-197609000-00003.

Cong, L., Ping, D., Daochun, C., \& Lin, C. (2015). Depression and associated factors in the elderly cadres in Fuzhou, China: A community-based study. International Journal of Gerontology, 9(1), 29-33.Available at: https://doi.org/10.1016/j.ijge.2015.02.001. 
Dahany, M. M., Dramé, M., Mahmoudi, R., Novella, J.-L., Ciocan, D., Kanagaratnam, L., \& Jolly, D. (2014). Factors associated with successful aging in persons aged 65 to 75 years. European Geriatric Medicine, 5(6), 365-370.Available at: https://doi.org/10.1016/j.eurger.2014.09.005.

Feng, Q., Yeung, W. J., Wang, Z., \& Zeng, Y. (2019). Age of retirement and human capital in an aging China, $2015-2050$. European Journal of Population, 35(1), 29-62.Available at: https://doi.org/10.1007/s 10680-018-9467-3.

Fone, D., Frank, D., Keith, L., Gareth, W., John, W., \& Stephen, P. (2007). Does social cohesion modify the association between area income deprivation and mental health? A multilevel analysis. International Journal of Epidemiology, 36, 338345.Available at: https://doi.org/10.1093/ije/dymo04.

Golja, K., Ana, M. D., \& Voyko, K. (2020). Cognitive reserve and depression predict subjective reports of successful aging. Archives of Gerontology and Geriatrics, 90, 104137.Available at: https://doi.org/10.1016/j.archger.2020.104137.

Grey, I., Teresa, A., Justin, T., Ahmad, S., Pia, T., \& Rudy, A.-H. (2020). The role of perceived social support on depression and sleep during the COVID-19 pandemic. Psychiatry Research, 293, 113452.Available at: https://doi.org/10.1016/j.psychres.2020.113452.

Hadjicharalambous, D., \& Demetriou, L. (2020). The quality of the parent-child relationship and children's family, school and social competences in Cyprus. International Journal of Social Sciences Perspectives, 7(1), 22-33.Available at: https://doi.org/10.33094/7.2017.2020.71.22.33.

Han, K.-M., Han, C., Shin, C., Jee, H.-J., An, H., Yoon, H.-K., \& Kim, S.-H. (2018). Social capital, socioeconomic status, and depression in community-living elderly. Journal of Psychiatric Research, 98, 133-140.Available at: https://doi.org/10.1016/j.jpsychires.2018.01.002.

Haseda, M., Kondo, N., Takagi, D., \& Kondo, K. (2018). Community social capital and inequality in depressive symptoms among older Japanese adults: A multilevel study. Health \& Place, 52, 8-17.Available at: https://doi.org/10.1016/j.healthplace.2018.04.010.

Hoyl, M. T., Alessi, C. A., Harker, J. O., Josephson, K. R., Pietruszka, F. M., Koelfgen, M., \& Rubenstein, L. Z. (1999). Development and testing of a five-item version of the geriatric depression scale. Journal of the American Geriatrics Society, 47(7), 873-878.Available at: https://doi.org/10.1111/j.1532-5415.1999.tbo3848.x.

Ji, L., Xiaoxia, Q., Yaru, J., Huaxin, S., Xinyi, L., \& Cuili, W. (2020). Age differences in the relationship between frailty and depression among community-dwelling older adults. Geriatric Nursing, 41, 485-489.Available at: https://doi.org/10.1016/j.gerinurse.2020.01.021.

Kamen, C., Cosgrove, V., McKellar, J., Cronkite, R., \& Moos, R. (2011). Family support and depressive symptoms: A 23-year follow-up. Journal of Clinical Psychology, 67(3), 215-223.

Landstedt, E., Ylva, B. A., Malin, E., \& Anne, H. (2016). Disentangling the directions of associations between structural social capital and mental health: Longitudinal analyses of gender, civic engagement and depressive symptoms. Social Science E Medicine, 163, 135-143.Available at: https://doi.org/10.1016/j.socscimed.2016.07.005.

Lei, X., Xiaoting, S., John, S., Peng, Z., \& Yaohui, Z. (2014). Depressive symptoms and SES among the mid-aged and elderly in China: Evidence from the China health and retirement longitudinal study national baseline. Social Science \& Medicine, 120, 224-232.Available at: https://doi.org/10.1016/j.socscimed.2014.09.028.

Li, Q., Zhou, X., Ma, S., Jiang, M., \& Li, L. (2017). The effect of migration on social capital and depression among older adults in China. Social Psychiatry and Psychiatric Epidemiology, 52(12), 1513-1522.Available at: https://doi.org/10.1007/s00127017-1439-0.

Li, T.-r., \& Hou, Y.-b. (2012). Psychological structure and psychometric validity of the confucian coping. Journal of Educational Science of Hunan Normal University, 11(3), 16-18.

Li, C., Jiang, S., Li, N., \& Zhang, Q. (2018). Influence of social participation on life satisfaction and depression among Chinese elderly: Social support as a mediator. Journal of Community Psychology, 46(3), 345-355.Available at: https://doi.org/10.1002/jcop.21944. 
Lihua, Z., Gui, C., Yanghua, J., Liqiong, L., \& Jian, C. (2017). Self-compassion and confucian coping as a predictor of depression and anxiety in impoverished Chinese undergraduates. Psychological Reports, 120(4), 627-638.Available at: https://doi.org/10.1177/0033294117700857.

Liu, D., Xi, J., Hall, B. J., Fu, M., Zhang, B., Guo, J., \& Feng, X. (2020). Attitudes toward aging, social support and depression among older adults: Difference by urban and rural areas in China. Journal of Affective Disorders, 274, 85-92.Available at: https://doi.org/10.1016/j.jad.2020.05.052.

McDonald, A., Thompson, A. J., Perzow, S. E., Joos, C., \& Wadsworth, M. E. (2020). The protective roles of ethnic identity, social support, and coping on depression in low-income parents: A test of the adaptation to poverty-related stress model. Journal of Consulting and Clinical Psychology, 88(6), 504-515.Available at: https://doi.org/10.1037/ccpo000477.

McNeish, D., \& Wolf, M. G. (2021). Dynamic fit index cutoffs for confirmatory factor analysis models. Psychological Methods, 26, 1-29.Available at: https://doi.org/10.1037/met0000425.

Murayama, H., Nishi, M., Nofuji, Y., Matsuo, E., Taniguchi, Y., Amano, H., \& Shinkai, S. (2015). Longitudinal association between neighborhood cohesion and depressive mood in old age: A Japanese prospective study. Health \& Place, 34, 270278.Available at: https://doi.org/10.1016/j.healthplace.2015.05.015.

Murray, C. B. (2015). Culture as a determinant of mental health. International Encyclopedia of the Social \& Behavioral Sciences, 5 , 570-575.Available at: https://doi.org/10.1016/B978-0-08-097086-8.14011-5.

Nakagomi, A., Shiba, K., Hanazato, M., Kondo, K., \& Kawachi, I. (2020). Does community-level social capital mitigate the impact of widowhood \& living alone on depressive symptoms?: A prospective, multi-level study. Social Science \&̊ Medicine, 259, 113140.Available at: https://doi.org/10.1016/j.socscimed.2020.113140.

National Bureau of Statistics of China. (2021). Statistical communiqué of the people's Republic of China on the 2020 National $\begin{array}{lllll}\text { Economic and } & \text { Social } & \text { Development. }\end{array}$ http://www.stats.gov.cn/english/PressRelease/202102/t20210228_1814177.html.

Park, M.-J. (2017). Impact of social capital on depression trajectories of older women in Korea. Aging $\mathfrak{F}^{2}$ Mental Health, 21(4), 354-361.Available at: https://doi.org/10.1080/13607863.2015.1088511.

Preacher, K. J., \& Hayes, A. F. (2008). Asymptotic and resampling strategies for assessing and comparing indirect effects in multiple mediator models. Behavior Research Methods, 4O(3), 879-891.Available at: https://doi.org/10.3758/brm.40.3.879.

Putnam, R. D. (1993). The prosperous community: Social capital and public life. American Prospects, 4(13), 35-42.

Rodgers, J., Valuev, A. V., Hswen, Y., \& Subramanian, S. (2019). Social capital and physical health: An updated review of the literature for 2007-2018. Social Science \& Medicine, 236, 112360.Available at: https://doi.org/10.1016/j.socscimed.2019.112360.

Rong, J., Ge, Y. H., Meng, N. N., Xie, T. T., \& Ding, H. (2020). Meta-analysis of the prevalence of depression in the elderly in China from 2010 to 2019. Chinese Journal of Evidence-based Medicine, 20, 26-31.Available at: https://doi.org/10.7507/1672-2531.201908088.

Rung, A. L., Symielle, G., William, T. R., Edward, J. T., \& Edward, S. P. (2017). Untangling the disaster-depression knot: The role of social ties after Deepwater Horizon. Social Science \& Medicine, 177, 19-26.Available at: https://doi.org/10.1016/j.socscimed.2017.01.041.

Saito, M., Kondo, N., Aida, J., Kawachi, I., Koyama, S., Ojima, T., \& Kondo, K. (2017). Development of an instrument for community-level health related social capital among Japanese older people: The JAGES project. Journal of Epidemiology, 27(5), 22 1-227.Available at: https://doi.org/10.1016/j.je.2016.06.005.

Schumacker, R. E., \& Richard, G. L. (2010). A beginner's guide to structural equation modeling (3rd ed., pp. 85-120). New York: Psychology Press.

Shen, Y. (2014). Community building and mental health in mid-life and older life: Evidence from China. Social Science $\mathcal{E}^{2}$ Medicine, 107, 209-2 16.Available at: https://doi.org/10.1016/j.socscimed.2013.12.023. 
Shrestha, S., Shibanuma, A., Poudel, K. C., Nanishi, K., Koyama Abe, M., Shakya, S. K., \& Jimba, M. (2019). Perceived social support, coping, and stigma on the quality of life of people living with HIV in Nepal: A moderated mediation analysis. AIDS Care, 31(4), 413-420.Available at: https://doi.org/10.1080/09540121.2018.1497136.

Thoits, P. A. (1986). Social support as coping assistance. Journal of Consulting and Clinical Psychology, 54(4), 416-423.Available at: https://doi.org/10.1037/0022-006x.54.4.416.

Trouillet, R., Gana, K., Lourel, M., \& Fort, I. (2009). Predictive value of age for coping: The role of self-efficacy, social support satisfaction and perceived stress. Aging and Mental Health, 13(3), 357-366.Available at: https://doi.org/10.1080/13607860802626223.

Vanoh, D., Shahar, S., Yahya, H. M., \& Hamid, T. A. (2016). Prevalence and determinants of depressive disorders among community-dwelling older adults: Findings from the towards useful aging study. International Journal of Gerontology, $10(2), 81-85$. Available at: https://doi.org/10.1016/j.ijge.2016.02.001.

Wang, J., \& Zhao, X. (2012). Family functioning and social support for older patients with depression in an urban area of Shanghai, China. Archives of Gerontology and Geriatrics, 55, 574-579.Available at: https://doi.org/10.1016/j.archger.2012.06.011.

Wang, R., Feng, Z., Liu, Y., \& Lu, Y. (2020). Relationship between neighbourhood social participation and depression among older adults: A longitudinal study in China. Health \& Social Care in the Community, 28(1), 247-259.Available at: https://doi.org/10.1111/hsc. 12859 .

Wu, Q. B., Bill, T., \& Holly, M. (2012). Social capital, family support, resilience and educational outcomes of Chinese migrant children. British Journal of Social Work, 44, 636-656.Available at: https://doi.org/10.1093/bjsw/bcs139.

Yamaguchi, M., Inoue, Y., Shinozaki, T., Saito, M., Takagi, D., Kondo, K., \& Kondo, N. (2019). Community social capital and depressive symptoms among older people in Japan: A multilevel longitudinal study. Journal of Epidemiology, 29(10), 363-369.Available at: https://doi.org/10.2188/jea.je20180078.

Zimet, G. D., Suzanne, S. P., Gordon, K. F., Sidney, W., \& Karen, A. B. (1990). Psychometric characteristics of the multidimensional scale of perceived social support. Journal of Personality Assessment, 55, 610-617.Available at: https://doi.org/10.1080/00223891.1990.9674095.

Views and opinions expressed in this article are the views and opinions of the author(s), Humanities and Social Sciences Letters shall not be responsible or answerable for any loss, damage or liability etc. caused in relation to/arising out of the use of the content. 